\title{
Convocatorias
}

\section{THE EUROPEAN ASSOCIATION FOR THE HISTORY OF MEDICINE AND HEALTH}

The European Association for the History of Medicine and Health (EAHMH) has been created recently, after several meetings at which medical historians from all over Europe and North America discussed the aims, objects, and organisation of such a new scientific society. All agreed on the need for a high level academic forum in Europe, at which scholars interested in the history of medicine and health, including historians of medicine and other medical specialties as well as demographers, social historians, social anthropologists, could meet and exchange ideas. The purpose of the Association is also the education of the public in the historical perspective of problems of medicine, health and disease.

The official seat of the Association is at the Universite Louis Pasteur, Strasbourg, and its first officers are as follows:

President: Prof. U. Tröhler (Göttingen);

Secretary/Treasurer: Prof. C. Debru (Strasbourg);

Members of the Council: Prof. H. Beukers (Leiden), B. Lindskog (Copenhagen); and the President of the Université Louis Pasteur (or his deputy).

The Association will hold a Congress every two years, and will also endeavour to set up various networks of interest groups to disseminate news and information. The first Congress will take place in Göttingen in September 1993. Contact Prof. U. Tröhler, Institut für Geschichte der Medizin, Humboldtallee 11, D - 3400 Göttingen.

There is a scientific board responsible for organising the Congresses and for promoting these scientific networks. Its members are: Dr. John Woodward (Sheffield), Chairman; Profs. Tröhler, Debru, Beukers; Profs. B. Fantini (Geneva and Naples), J. Geyer-Kordesch (Glasgow), M. J. Imbault-Huart (Paris), R. Jütte (Stuttgart)- E. Seidler (Freiburg i. Br.), J. Sundin (Linköping).

Membership in the Association is not confined to scholars living or working in Europe. Beginning in 1992, the annual subscription will be 150 French Francs, in exchange of which members will receive an annual newsletter as well as the right to purchase at reduced cost the proceedings of the Congresses and other Association publications. Contact Prof. C. Debru, Centre Européen d'Histoire de la Médecine, Faculté de Médecine, 4, rue Kirschleger, 67085 Strasbourg Cedex, France. 


\section{ASOCIACIÓN EUROPEA DE HISTORIA DE LA MEDICINA Y DE LA SALUD}

Recientemente se ha fundado la Asociación Europea de Historia de la Medicina y de la Salud (E.A.H.M.H.), luego de celebradas distintas reuniones en las que historiadores de toda Europa y Norteamérica han discutido el objeto, los fines y la organización de dicha nueva sociedad. Existió consenso sobre la conveniencia de que existiera un foro académico de alto nivel en Europa, donde los estudiosos interesados en la historia de la salud y la medicina, esto es, historiadores de la medicina y cultivadores de otras especialidades médicas, así como demógrafos, historiadores sociales o antropólogos sociales, pudieran encontrarse e intercambiar ideas. También es propósito de la Asociación ofrecer a la opinión pública la perspectiva histórica sobre problemas de la medicina, la salud y la enfermedad.

La sede oficial de la Asociación es la Universidad Louis Pasteur de Estrasburgo y sus cargos directivos son los siguientes:

Presidente: Prof. U. Tröhler (Göttingen);

Secretario/Tesorero: Prof. C. Debru (Estrasburgo);

Vocales del Consejo: Prof. H. Beukers (Leiden), B. Lindskog (Copenhague) y el Rector de la Universidad Louis Pasteur o persona en quien delegue.

La Asociación se propone la organización bianual de un Congreso, así como facilitar los contactos entre grupos de trabajo con los mismos intereses para facilitar la diseminación de información. El primer Congreso tendrá lugar en Göttingen en septiembre de 1993. Para más información, diríjanse al Prof. U. Tröhler, Institut für Geschichte der Medizin, Hurnboldtallee 11, D-3400, Göttingen.

Existe un Consejo científico responsable de la organización de los Congresos y de la promoción de las redes de investigación mencionadas, compuesto por: Dr. John Woodward (Sheffield), que lo dirige; Profs. Tröhler, Debru, Beukers, B. Fantini (Ginebra y Nápoles), J. Geyer-Kordesch (Glasgow), M. J. Imbault-Huart (París), R. Jütte (Stuttgart), E. Seidler (Freiburg i. B.) y J. Sundin (Linköping).

La pertenencia a la Asociación no está limitada por razones geográficas. La cuota anual, a partir de 1992, será de 150 francos franceses, a cambio de la cual los socios recibirán una newsletter anual así como el derecho a adquirir a costo reducido las actas de los Congresos y restantes publicaciones de la Asociación. Para más información, ponerse en contacto con el Prof. C. Debru, Centre Européen d'Histoire de la Médecine, Faculté de Médecine, 4, rue Kirschleger, 67085 Strasbourg Cedex, Francia. 